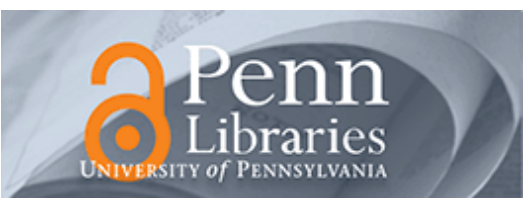

University of Pennsylvania

ScholarlyCommons

\title{
Application of a new Lyapunov function to global adaptive attitude tracking
}

Daniel E. Koditschek

University of Pennsylvania, kod@seas.upenn.edu

Follow this and additional works at: https://repository.upenn.edu/ese_papers

\section{Recommended Citation}

Daniel E. Koditschek, "Application of a new Lyapunov function to global adaptive attitude tracking", . December 1988.

Copyright 1988 IEEE. Reprinted from Proceedings of the 27th IEEE Conference on Decision and Control, Volume 1, 1988, pages 63-68.

This material is posted here with permission of the IEEE. Such permission of the IEEE does not in any way imply IEEE endorsement of any of the University of Pennsylvania's products or services. Internal or personal use of this material is permitted. However, permission to reprint/republish this material for advertising or promotional purposes or for creating new collective works for resale or redistribution must be obtained from the IEEE by writing to pubs-permissions@ieee.org. By choosing to view this document, you agree to all provisions of the copyright laws protecting it.

NOTE: At the time of publication, author Daniel Koditschek was affiliated with Yale University. Currently, he is a faculty member in the Department of Electrical and Systems Engineering at the University of Pennsylvania.

This paper is posted at ScholarlyCommons. https://repository.upenn.edu/ese_papers/392

For more information, please contact repository@pobox.upenn.edu. 


\title{
Application of a new Lyapunov function to global adaptive attitude tracking
}

\author{
Abstract \\ The introduction of "error coordinates" and a "tracking potential" on the rotations affords a global \\ nonlinear version of inverse dynamics for attitude tracking. The resulting algorithm produces "almost \\ global" asymptotically exact tracking: this convergence behavior is as strong as the topology of the phase \\ space can allow. A new family of strict global Lyapunov functions for mechanical systems is applied to \\ achieve an adaptive version of the inverse dynamics algorithm in the case that the inertial parameters of \\ the rigid body are not known a priori. The resulting closed loop adaptive system is shown to be stable, \\ and the rigid body phase errors are shown to converge to the limit trajectories of the non-adaptive \\ algorithm.
}

\section{Comments}

Copyright 1988 IEEE. Reprinted from Proceedings of the 27th IEEE Conference on Decision and Control, Volume 1, 1988, pages 63-68.

This material is posted here with permission of the IEEE. Such permission of the IEEE does not in any way imply IEEE endorsement of any of the University of Pennsylvania's products or services. Internal or personal use of this material is permitted. However, permission to reprint/republish this material for advertising or promotional purposes or for creating new collective works for resale or redistribution must be obtained from the IEEE by writing to pubs-permissions@ieee.org. By choosing to view this document, you agree to all provisions of the copyright laws protecting it.

NOTE: At the time of publication, author Daniel Koditschek was affiliated with Yale University. Currently, he is a faculty member in the Department of Electrical and Systems Engineering at the University of Pennsylvania. 


\title{
Application of a New Lyapunov Function to Global Adaptive Attitude Tracking
}

\author{
Daniel E. Koditschek ${ }^{1}$ \\ Center for Systems Science \\ Yale University, Department of Electrical Engineering
}

\begin{abstract}
The introduction of "error coordinates" and a "tracking potential" on the rotations affords a global nonlinear version of inverse dynamics for attitude tracking. The resulting algorithm produces "almost global" asymptotically exact tracking: this convergence behavior is as strong as the topology of the phase space can allow. A new family of strict global Lyapunov functions for mechanical systems is applied to achieve an adaptive version of the inverse dynamics algorithm in the case that the inertial parameters of the rigid body are not known à priori. The resulting closed loop adaptive system is shown to be stable, and the rigid body phase errors are shown to converge to the limit trajectories of the non-adaptive algorithm.
\end{abstract}

\section{Introduction}

This paper presents a sample application of a new family of strict global Lyapunov functions for mechanical systems on finite dimensional Riemannian manifolds. This new class of Lyapunov functions was presented in a recent conference paper [10] wherein application was made to a mechanical system on a Euclidean vector space. There, the only nonlinearity in the dynamics arose via the kinetic energy. In this paper we consider the problem of tracking a desired signal on a Non-Euclidean space - the Lie group $S O(3)$ - via a completely actuated mechanical system with all states directly available. Neither the dynamics nor the state space are linear.

A "mechanical system" is a Lagrangian dynamical system whose geodesics arise from a Riemannian metric defined by the kinetic energy of a (possibly mutually constrained) set of rigid bodies. This paper concerns the dynamics of a single rigid body. We take the word "tracking" to mean the asymptotic coincidence of the output of a controlled system with a perfectly known but entirely arbitrary reference signal. For a linear time invariant system, the only means of accomplishing such a task is by recourse to inverse dynamics plant stabilization via feedback; plant inversion via a precompensating filter - and this paper incorporates the same methodology in a nonlinear context. Since the removal of any structural information about the class of reference signals translates into the reliance upon exact plant parameter values, it seems incumbent upon the designer to deploy an adaptive version of the algorithm if possible. Thus, as its central contribution, this paper offers an adaptive inverse dynamics algorithm for a single rigid body powered by three independent actuators capable of delivering any desired force in the "wrench space" of the body, $T^{*} S O(3)$, assuming perfect state information.

Since our system is completely actuated, we are free to prescribe arbitrary acceleration. Thus, from the point of view of velocity control the problem admits a trivial implementation of the global exact linearization techniques which have become so popular in the nonlinear control literature. Evidently, there is little new to be said regarding the construction of time invariant feedback control laws, $u: \mathbb{R}^{3} \rightarrow \mathbb{R}^{3}$, which cause the actual angular velocity, $a(t) \in \mathbb{R}^{3}$, to track a desired angular velocity, $d(t) \in \mathbb{R}^{3}$ when, as in the present

${ }^{1}$ This work was supported in part by the National Science Foundation under grant no. DMC-8505160, and in part by a National Science Foundation Presidential Young Investigator Award. case, the control system is equivalent to $\dot{a}=u$. However, the problem addressed above is quite different: we are given a desired attitude trajectory, $D(t) \in S O(3)$, and asked to construct a time invariant controller which causes the actual attitude, to asymptotically approach $D(t)$ from any initial configuration, $A \in S O(3)$.

So different is this problem from the trivial double integrator tracking problem that it is unsolvable as posed in that context. For, consider the particular case that $D(t)=D^{*}$ is some constant point. We seek a controller which makes that point (at zero angular velocity) a global attractor of the closed loop dynamics. Now the domain of attraction of an attracting point is homeomorphic to some Euclidean vector space [3]. But the state space of our mechanical system - the cross product $T S O(3) \approx S O(3) \times \mathbb{R}^{3}$ - is clearly not homeomorphic to any Euclidean vector space. Thus, it would be impossible for our closed loop system to bring all initial conditions to the desired attitude. Evidently, the control system arising from a single rigid body is not globally linearizable by any technique since its state space is not a vector space. Our problem statement must be refined.

Since $S O(3)$ is a compact odd dimensional manifold without boundary, its Euler characteristic is zero [7]. It follows from the Theorem of Hopf [12] that any nondegenerate vector field on $S O(3)$ with an attracting equilibrium state has at least one other singularity which, if it is the only additional equilibrium state, must be totally unstable. Excepting the complement of some open dense set - in this case, the repelling point - trajectories of such a vector field are guaranteed to asymptotically approach the attracting point. Thus, although topological obstructions preclude a globally asymptotically stable system, a practically equivalent formulation which respects the underlying topology of the problem may be attainable. Say that a dynamical system is almost globally asymptotically stable if all trajectories starting in some open dense subset of the state space tend asymptotically to a specified stable equilibrium state. This we take as the criterion of convergence for our tracking algorithms on $S O(3)$.

The paper is organized as follows. In Section 2 we first review certain properties of $S O(3)$ and its Lie algebra to arrive at "error coordinates", then present a global version of Lord Kelvin's century old result on energy dissipation leading to the notion of a tracking potential function. Appropriate error dynamics having been targeted, the obvious generalization of the inverse dynamics algorithm is shown to achieve them, with almost global convergence in consequence. Namely, all trajectories starting from some open dense subset of the possible initial errors achieve asymptotically exact tracking; tra jectories starting from a nowhere dense set in the phase space approach one of three other isolated "antipodal" motions. In Section 3 we showcase the utility of the new family of strict Lyapunov function presented in [10] by considering the adaptive version of the previous tracking algorithm. This version of the problem arises when there is uncertainty regarding the inertia matrix of the rigid body. After reviewing and slightly extending our earlier constructive results for general mechanical systems, we implement a particular choice from this family of Lyapunov functions picked to admit a straightforward analogy between linear time invariant adaptive control algorithms and the present setting. The resulting closed loop adaptive system is shown to be stable, and gives rise to trajectories whose projections onto the state space approach one of the four (one correct and 
three spurious isolated "antipodal") limit motions observed in the previous section. Presumably, an appropriate notion of "persistent excitation" would lead to guarantees concerning the practical impossibility of ever ending up tracking one of the spurious motions, but this question is deferred to a future paper.

In the interest of brevity, a variety of technical definitions and results are used with little explanation. An attempt has been made to provide a precise reference to the literature in such cases. Certain of the new technical results are merely stated: the reader is referred to the author's two recent reports $[8,9]$ for detailed proofs.

\section{Inverse Dynamics on the Spatial Rotations}

In the linear time invariant setting, inverse dynamics amounts to the use of a precompensator to make the errors between the plant state and reference derivatives satisfy an asymptotically stable linear time invariant dynamical system. For example, after stabilizing

$$
\ddot{x}=u(t),
$$

with $u=-K_{1} x-K_{2} \dot{x}+v$, we may cause the plant to track an arbitary reference signal, $r$, by pre-filtering, $v=\ddot{r}-K_{1} r-K_{2} \dot{r}$, since this results in globally asymptotically stable error dynamics,

$$
\ddot{e}=-K_{1} e-K_{2} \dot{e} .
$$

When the state space is not a Euclidean vector space, we must first determine a suitable notion of "error", next find a globally asymptotically stable (or almost globally asymptotically stable in the sense defined in the introduction) vector field that we would would wish to govern the error dynamics, and only then look for a suitable precompensator for the input signal. In Section 2.1 we review a number of useful facts about the configuration space of the rigid body and end up with a natural choice of error coordinates. In Section 2.2, we introduce the class of mechanical systems and describe their relationship to gradient dynamics, motivating the choice of a tracking potential function in Section 2.3. Finally, in Section 2.4 we present the inverse dynamics algorithm and show that it achieves almost global asymptotically exact tracking.

\subsection{Error Coordinates on $S O(3)$}

The configuration space of a rigid body is the group of rigid transformations, $S O(3) \times \mathbb{R}^{3}$. If we are concerned only with the attitude of a rigid body, then it suffices to treat $S O(3)$ alone, which we now identitfy with a subset of $\mathbb{R}^{9}$,

$$
S O(3) \triangleq\left\{R \in \mathbb{R}^{3 \times 3}: R^{\mathrm{T}} R=I \text { and }|R|=1\right\} .
$$

This Lie group has as its Lie algebra the skew symmetric matrices,

$$
\text { so(3) }=\text { skew } \triangleq\left\{J \in \mathbb{R}^{3 \times 3}: J+J^{\mathrm{T}}=0\right\},
$$

which is isomorphic to $\mathbb{R}^{3}$ according to the linear bijection

$$
J: w \mapsto\left[\begin{array}{ccc}
0 & -w_{3} & w_{2} \\
w_{3} & 0 & -w_{1} \\
-w_{2} & w_{1} & 0
\end{array}\right] .
$$

The vector space of three by three matrices is the direct sum,

$$
\mathbb{R}^{3 \times 3}=\text { sym } \oplus \text { skew, }
$$

of the symmetric and skew-symmetric matrices. Thus, we may define a unique "pseudo-inverse" for $J$ whose domain is extended to all of $\mathbb{R}^{3 \times 3}$ by projection onto the linear subspace, skew.

$$
J^{\dagger}(A) \triangleq J^{-1}\left(A-A^{\mathrm{T}}\right)
$$

The maps $J^{-1}$ and $J^{\dagger}$ have distinct domains, and must not be confused. On the other hand, we will be sloppy and not distinguish between the version of the linear map $J$ whose range is skew and the version whose range is $\mathbb{R}^{3 \times 3}$.

The natural inner product on the vector space $\mathbb{R}^{3 \times 3}$ is

$$
\left(M_{1} \mid M_{2}\right) \triangleq \frac{1}{2} \operatorname{tr}\left\{M_{1} M_{2}^{\mathrm{T}}\right\} .
$$

Direct computation reveals that $J$ is an isometry between $\mathbb{R}^{3}$ with its Euclidean norm, $\|w\|^{2}=w^{\mathrm{T}} w$, and skew with the norm corresponding to this inner product. Note, as well, that sym is the orthogonal complement of skew with respect to this inner product. Finally, the norm associated with $(\cdot \mid \cdot)$ defines a metric on the group $S O(3)$ after composition with a suitable comparison function. ${ }^{1}$

Proposition 2.1 ( [8] ) Consider the smooth comparison function

$$
\xi \in \mathcal{K}_{\infty}[[0, \pi],[0,4]]: \chi \mapsto 2(1-\cos \chi) .
$$

The composition of its inverse with the natural Euclidean norm of $\mathbb{R}^{3 \times 3}$

$$
\rho\left(R_{1}, R_{2}\right) \triangleq \xi^{-1} \circ\left(R_{1}-R_{2} \mid R_{1}-R_{2}\right),
$$

defines a metric on $S O(3)$

On any Lie group, we may take the differential of left (or right) inverse translation and this is the canonical means of identifying left (or right) invariant vector fields with the Lie algebra. Thus,

$$
T_{R} S O(3)=\left\{R J(w) \in \mathbb{R}^{3 \times 3}: w \in \mathbb{R}^{3}\right\},
$$

is identified once and for all with $s o(3)=$ skew $\approx \mathbb{R}^{3}$, and we may take the tangent bundle to be the set of pairs

$$
T S O(3)=\left\{(R, r): R \in S O(3), r \in \mathbb{R}^{3}\right\} .
$$

Now, given two curves, $(A, a)(t),(D, d)(t) \in T S O(3)$, assumed to be second order [1, Def. II.3.5.12] - e.g. $\dot{A}(t)=A J(a(t))$ - we define their "error" to be the tangent map induced by left inverse translation to the identity,

$$
(E, e)(t) \triangleq\left(D^{\mathrm{T}} A, a-A^{\mathrm{T}} D d\right)(t),
$$

preserving the second order property, $\dot{E}(t)=E J(e(t))$.

\subsection{Gradient and Lagrangian Dynamics}

The geometry of classical physics has been extensively studied for decades, and recent years have witnessed the publication of numerous expository texts containing the background material required for the present paper. We find it most useful to appeal to the notation and presentation of the excellent text by Abraham and Marsden [1]. In addition, we will adopt the language of vector bundle morphisms [7][Ch. 4.1], $\mathcal{M}^{r}[\mathcal{X}, \mathcal{Y}]$ between two vector bundles, $\mathcal{X}, \mathcal{Y}$, over some manifold, $\mathcal{J}$. For example, a Riemannian metric on $\mathcal{J}$, is induced by every "positive definite morphism", $M \in \mathcal{M}^{\top}\left[T \mathcal{J}, T^{*} \mathcal{J}\right]$.

Non-degenerate gradient vector fields on the configuration space have particularly nice limiting properties, as summarized by the fol lowing result.

${ }^{1}$ The comparison functions, the group $\mathcal{K}_{r}\left(\mathcal{I}_{1}, \mathcal{I}_{2}\right)$, of monotone increasing $C^{r}$ diffeomorphisms between two real intervals appears extensively in the engineering stability literature [6], as well as in this paper. Some properties are reviewed in $[8,9]$ 
Proposition 2.2 ( [9] ) Let $\varphi$ be a continuously differentiable Morse function on the compact Riemannian manifold, $\mathcal{J}$. Suppose that $\operatorname{grad} \varphi$ is transverse and directed away from the interior of $\mathcal{J}$ on any boundary of that set. Then the negative gradient flow has the following properties:

\section{1. $\mathcal{J}$ is a positive invariant set;}

2. the positive limit set of $\mathcal{J}$ consists of the critical points of $\varphi$;

3. there is a dense open set $\tilde{J} \subset \mathcal{J}$ whose limit set consists of the local minima of $\varphi$,

Still more important for the present purposes is the natural "lifting" of gradient vector fields (considered as potential fields) into Lagrangian vector fields, $f_{\lambda}$, on $T \mathcal{J},[1, \mathrm{Ch}$. II.3.7]. By a dissipative mechanical system we mean the dynamics associated with the vector field

$$
f \triangleq f_{\lambda}+f_{d}
$$

where $f_{d}$ is a dissipative vector field [1, Def. II.3.7.16]. The crucial result we require for this section of the paper is that the limit behavior of a dissipative Lagrangian system is a "copy" of the limit behavior of its constituent potential energy gradient field. This will be recognized as a global version of Lord Kelvin's century old result [14] concerning the dissipation of total energy on $T \mathcal{J}$

$$
\eta(w) \triangleq \frac{1}{2}\langle w \mid w\rangle+\varphi \circ \tau(w),
$$

under the flow of $f$.

Theorem 1 ( [9] ) Let $\varphi$ be a twice differentiable Morse function on the compact Riemannian manifold, $\mathcal{J}$. If $\mathcal{J}$ has a boundary, then let $\varphi$ attain its maximum, $\eta_{0}$, over $\mathcal{J}$, exactly on that boundary. The set of "bounded total energy" states of $\eta$ (2),

$$
\mathcal{E}^{\eta_{0}} \triangleq\left\{v \in T \mathcal{J}: \eta \leq \eta_{0}\right\},
$$

is a positive invariant set of any dissipative mechanical system (1) whose positive limit set consists of those points in the zero section of $T \mathcal{J}$ identified with a critical point of the potential function. All initial conditions within $\mathcal{E}^{\eta_{0}}$ excluding a nowhere dense set tend toward a point in the zero section of $T \mathcal{J} \cap \mathcal{E}^{\eta_{0}}$ identified with a local minimum of $\varphi$.

\subsection{An Attitude Tracking Potential Function}

We are now ready to search for a potential function on $S O(3)$ whose lift into the physical Lagrangian system of the actuated rigid body will define almost globally asymptotically stable error dynamics. According to Theorem 1 , we need merely ensure that $\varphi$ is a Morse function with a unique minimum.

In a very nice report, Meyer [11] attempted to generalize PD techniques to the global control of spacecraft attitude. His point of view is very close to the spirit of this paper, and, in some sense, this application might be seen as a continuation and extension of that earlier work. Meyer chose for his potential law on $S O(3)$ the metric, $\rho$, itself. This will not suffice for the present purposes since, as seen in Proposition $2.1, \rho$ is a composition with the trace function on skew. The latter is unfortunately not a Morse function since it has a critical point at every symmetric rotation: the symmetric rotations - an embedding of real projective two-space, $\mathbb{R} \mathbb{P}^{2}$, in $S O(3)$ - comprise a connected set; the critical points are not isolated [5]. Instead we will use a "modified trace" function according to the following result of Marsden and collaborators.
Lemma 2.3 (Chillingworth, Marsden, and Wan [4] ) If $P \in$ sym has distinct eigenvalues, $\pi_{1}, \pi_{2}, \pi_{3}$, and

$$
\left(\pi_{1}+\pi_{2}\right)\left(\pi_{1}+\pi_{3}\right)\left(\pi_{3}+\pi_{2}\right) \neq 0
$$

then there are exactly four rotations, $R \in S O(3)$ at which $P R \in$ sym These are exactly the critical points of

$$
(P \mid R)=\operatorname{tr}\{P R\}
$$

with Morse index specified by the number of positive eigenvalues of

$$
\overline{P R} \triangleq \operatorname{tr}\{R P\} I-P R
$$
$S O(3)$

We are thus led to define as a tracking potential function on

$$
\varphi(R) \triangleq \operatorname{tr}\{P(I-R)\}=(P \mid I-R) .
$$

If $P \in \mathrm{sym}^{+}$, a positive definite symmetric matrix, which we now assume, then the eigenvalue assumptions of the previous Lemma are assured. Since $d \varphi$ is a scalar multiple of Marsden's modified trace, $\varphi$ is also a Morse function with four critical points specified in the same fashion. Moreover, $\varphi$ takes its values on $\overline{\mathbb{R}^{+}}$, vanishing only at $R=I$. Thus, $\varphi$ is indeed a candidate tracking potential function on $S O(3)$. In fact, it is the best we can do as the following result indicates.

Proposition 2.4 ( [8] ) Any Morse function on SO(3) has at least four critical points.

To finish this section, we display the following computation.

Lemma 2.5 The action of the co-vector, $d \varphi$, on a tangent vector,

$$
w \in T_{R} S O(3)
$$

is given by $c^{\mathrm{T}} w$ where

$$
c=2 J^{\dagger}(P R)
$$

Proof: $\quad$ Consider a smooth curve, through $R$ whose tangent vector is $w$ at $t=0$,

$$
R(t)=R \exp \{t J(w)\} .
$$

The action of the co-vector field $d \varphi$ on $w$ is exactly

$$
\begin{aligned}
\left.\frac{d}{d t} \varphi(R)\right|_{t=0} & =-\operatorname{tr}\{P R J(w)\} \\
& =(P R \mid J(w)) \\
& =2 w^{\mathrm{T}} J^{\dagger}(P R)
\end{aligned}
$$

since sym is orthogonal to skew, and $J^{\dagger}$ is an isometry between (skew, $\left.(\cdot \mid \cdot)^{\frac{3}{2}}\right)$ and $\left(\mathbb{R}^{3},\|\cdot\|\right)$.

$\square$

Thus, the gradient vector field of $\varphi$ with respect to a Riemannian metric, $\left\langle w^{\mathrm{T}} \mid w\right\rangle \triangleq w^{\mathrm{T}} M w$ on $T S O(3)$ is

$$
\operatorname{grad} \varphi=2 M^{-1} J^{\dagger}(P R) .
$$

\subsection{Almost Global Inverse Dynamics}

Now suppose $(D(t), d(t))$ is a reference trajectory in $T S O(3)$. Suppose, moreover, that the rigid body has moment of inertia, $M$, so that its Lagrangian vector field on $\operatorname{TSO}(3)$ admits the expression (using body coordinates to obtain left translation back to so(3)) are given, for example, as in [2], by 


$$
\dot{a}=M^{-1}[u-J(a) M a],
$$

where $u \in s o(3)^{*} \approx \mathbb{R}^{3}$ is the control input in "wrench space".

Since

$$
\begin{aligned}
\dot{e} & =\dot{a}-E^{\mathrm{T}} \dot{d}-\left[E J\left(a-E^{\mathrm{T}} d\right)\right]^{\mathrm{T}} d \\
& =\dot{a}-E^{\mathrm{T}} \dot{d}+J(a) E^{\mathrm{T}} d,
\end{aligned}
$$

the feedback law

$$
\begin{aligned}
u_{I D} \triangleq & M\left(E^{\mathrm{T}} \dot{d}-J(a) E^{\mathrm{T}} d\right) \\
& +J(a) M E^{\mathrm{T}} d+J\left(E^{\mathrm{T}} d\right) M e-K_{2} e-\operatorname{grad} \varphi(E),
\end{aligned}
$$

results in the closed loop system

$$
\dot{a}=E^{\mathrm{T}} \dot{d}-J(a) E^{\mathrm{T}} d-M^{-1}\left[J(e) M e+K_{2} e+\operatorname{grad} \varphi(E)\right],
$$

or, in the "error coordinate system" of phase space, $\operatorname{TSO}(3)=S O(3) \times$ $\mathbb{R}^{3}$,

$$
\begin{aligned}
\dot{E} & =E J(e) \\
\dot{e} & =-M^{-1}\left[J(e) M e+K_{2} e+\operatorname{grad} \varphi(E)\right]
\end{aligned}
$$

Theorem 2 If $K_{2}$ is a positive definite symmetric matrix then all trajectories of (6) tend toward one of the four critical points of $\varphi$. A dense open set of initial conditions has its limit set at the minimum, $(E, e)=(I, 0)$.

Proof: This is a direct application of Theorem 1 using the information in Lemma 2.3 to determine the number and stability properties of the critical points of $\varphi$.

ㅁ

\section{Application of Strict Global Lyapunov Func- tions}

The new family of strict global Lyapunov functions introduced in [10] requires the same setup as in Section 2.2 with a few' extra assumptions. We now assume the existence of a second Riemannian metric on $\mathcal{J},\|\cdot\|$, which is uniformly equivalent to the kinetic energy metric, $\langle\cdot \mid \cdot\rangle^{\frac{1}{2}}$, in the sense that there exist two constants, $\kappa_{1}, \kappa_{2}$, such that for all $w \in T \mathcal{J}$,

$$
\kappa_{1}\|w\|_{q} \leq\langle w \mid w\rangle_{q}^{\frac{1}{2}} \leq \kappa_{2}\|w\|_{q}
$$

We similarly assume that the Riemannian connection, $\nabla$, generated by the kinetic energy gives rise to a covariant derivative which is bounded with respect to $\|\cdot\|$. It may be readily verified in the present application that

$$
\langle w \mid w\rangle \triangleq w^{\mathrm{T}} M w ; \quad\|w\| \triangleq \boldsymbol{w}^{\mathrm{T}} w
$$

are equivalent, and that the connection corresponding to $\langle\cdot \mid \cdot\rangle$ gives rise to a bounded covariant derivative. Finally, for the sake of technical simplicity in this paper, we will assume that the dissipative field, $f_{d}$, takes the form of Rayleigh damping [1, Exc. II.3.7A].

In the earlier work cited above, $[9,10]$, the author has introduced a new Lyapunov function formed from the addition of an "angle measurement" between the true and desired velocity, followed by a consequent rescaling of the total energy, $\eta$. We first review these results in the next section, and apply that general analysis to the case at hand in the following section.

\subsection{Previous Results and an Extension}

The Riemannian metric is a symmetric isomorphism in $\mathcal{M}^{\omega}\left[T \mathcal{J}, T^{*} \mathcal{J}\right]$. By choosing a morphism in the other direction one obtains an "angular comparison" between the true velocity and the desired potential co-vector field, as follows. Say that a morphism, $F \in \mathcal{M}^{\omega}\left[T^{*} \mathcal{J}, T \mathcal{J}\right]$, is a pre-metric if its symmetric part is a "positive definite" operator on each fiber. Given a a manifold, $\mathcal{J}$, with a Riemannian metric, $M$, on the tangent bundle, $\tau: T \mathcal{J} \rightarrow \mathcal{J}$, a pre-metric, $F$, and a scalar valued map, $\varphi \in C^{1}[\mathcal{J}, \mathbb{R}]$, define the potential angle map, $\alpha \in C^{1}[T \mathcal{J}, \mathbb{R}]$ by

$$
\alpha(v) \triangleq\langle F d \varphi \circ \tau(v) \mid v\rangle .
$$

Lemma 3.1 ( [10] ) The Lie derivative of the potential angle map, $\alpha$,along the dissipative mechanical system, $f,(1)$, is a scalar valued map on $T \mathcal{J}$ which may be expressed as

$$
L_{f} \alpha(v)=\langle B v \mid v\rangle-\left\langle F d \varphi \circ \tau(v) \mid K_{2} v\right\rangle-(d \varphi \cdot F d \varphi) \circ \tau(v) .
$$

where $B \in \mathcal{M}^{\omega}[T \mathcal{J}, T \mathcal{J}]$ denotes the morphism,

$$
B: v \mapsto \nabla_{v} F d \varphi \text {. }
$$

Since $L_{f} \alpha$ has a term which is negative definite on $\mathcal{J}$, it is intuitively reasonable to hope that a strict Lyapunov function might result from adding $\alpha$ to $\eta$. This idea works after an an appropriate rescaling of $\eta$ which serves to dominate the indefinite terms of $L_{f} \alpha$. In the sequel, we will make free use of the notion of lower and upper comparison functions, $\lambda_{\psi}, v_{\psi}$, for scalar valued maps, $\psi$, on $\mathcal{J}$, as well as lower and upper magnitude functions, $\nu_{M}, \mu_{M}$, for morphisms, $M$, over $T \mathcal{J}$, their associated scalar functions, $\lambda_{M}, v_{M}$, and their constant lower and upper bounds $-\overline{\nu_{M}}, \overline{\mu_{M}}$. These are defined in the appendix, and they are critical components in the construction of the new Lyapunov function.

Theorem 3 ( [10] ) Let $f$ be a dissipative mechanical system (1). For any valid potential angle map, $\alpha,(7)$, define the comparison function, $\gamma \in \mathcal{K}_{1}$,

$$
\begin{aligned}
& \gamma(\chi) \triangleq \gamma_{0} \chi+\gamma_{1} \cdot\left(v_{d \varphi}^{2} \circ \lambda_{\varphi}^{-1}\right)(\chi) \\
&+\gamma_{2} \cdot\left[\int_{0}^{\chi}\left(v_{(M B),} \circ \lambda_{\eta}^{-1}\right)(\sigma) d \sigma\right],
\end{aligned}
$$

where $\gamma_{i}$ are non-decresaing functions satisfying

$$
\begin{aligned}
& \gamma_{0}>1+\left(\frac{1}{4 \nu_{F_{s}} \overline{\overline{\nu_{K_{2}}}}}\right) v_{F}^{2} \mathrm{~T}_{M K_{2}} \circ \lambda_{\eta}^{-1} \\
& \gamma_{1}>\frac{1}{4 \overline{\overline{\nu M}}} v_{F^{\mathrm{T}}} \circ \lambda_{\eta}^{-1} \\
& \gamma_{2}>\frac{1}{\overline{\nu_{K_{2}}}} .
\end{aligned}
$$

Then

$$
\vartheta \triangleq \gamma \circ \eta+\alpha
$$

is a positive definite function which has a derivative along trajectories of $f$, bounded by

where

$$
\dot{\vartheta}=L_{f}(\vartheta) \leq-x^{\mathrm{T}} Q x
$$

$$
x(v) \triangleq\left[\begin{array}{c}
\|d \varphi \circ \tau\| \\
\|v\|
\end{array}\right]
$$

and $Q$ is the $2 \times 2$ positive definite matrix valued function

$$
Q(v) \triangleq\left[\begin{array}{cc}
\nu_{F_{s}} & \frac{1}{2} \mu_{F^{\mathrm{T}}} \mathrm{T}_{K_{2}} \\
\frac{1}{2} \mu_{F^{\mathrm{T}} \mathrm{T}_{M K_{2}}} & \overline{\nu_{K_{2}}} \gamma_{0} \circ \eta
\end{array}\right] .
$$

We will now offer a slight extension to this construction suitable to the problem at hand - adaptive control using $\vartheta$ in the parameter adjustment laws. If the metric morphism, $M$, is unknown, yet some portion of $\vartheta$ which depends upon it remains after the construction of the adjustment laws, then we obviously have a fatuous algorithm. It turns out that the derivative of the rescaling function, $\gamma^{\prime} \circ \eta$, remains 
a part of any adjustment law based upon presently understood methods of parameter adaptation. Thus, we must find conditions under which $\gamma$ is a multiple of the identity comparison function, $\gamma(\chi)=\bar{\gamma} \chi$, so that $\gamma^{\prime}$ will be the constant function $\bar{\gamma}$. We proceed to investigate this possibility.

Say that $\vartheta$ has unscaled energy if $\gamma$ is a multiple of the identity comparison function. Say that $\varphi$ is a uniform potential if the ratio

$$
\frac{\frac{d^{2} \varphi \circ c(t)}{d t^{2}}}{1+\left|\frac{d \varphi \circ(t)}{d t}\right|}
$$

is bounded for any smooth curve in the configuration space and if there can be found a lower comparison function for $\varphi, \lambda_{\varphi}$, and an upper comparison function for $\|d \varphi\|, v_{d \varphi}$ such that

$$
v_{d \varphi}^{2} \circ \lambda_{\varphi}
$$

is a scalar multiple of the identity map of $\mathcal{K}_{r}$.

Proposition 3.2 If $\varphi$ is a uniform potential function then there exists a a strict Lyapunov function, $\vartheta$, for $f$ with unscaled energy.

Proof: We must exhibit a morphism, $F$, for which the compositions in (8) are all multiples of the identity, and for which the gain inequalities (9) are satisfied by constants. Define the morphsim, $F$, to be

$$
\frac{1}{1+\|d \varphi\|} I,
$$

where $I: T^{*} \mathcal{J} \rightarrow T \mathcal{J}$ is the isomorphism which is isometric with respect to $\|\cdot\|$. It is shown in [8] that following the recipe for $\vartheta$ using this choice of $F$ leads to a function with unscaled energy.

\subsection{Adaptive Inverse Dynamics on $S O(3)$}

We now suppose that it is desired to implement (5) but that $M$ is unknown and the control is realized with an estimate, $\hat{M}$, which is to be tuned in response to the observed error. Namely, we apply the control law $u_{I D}+n(t)$ where

$$
n(t)=\tilde{M}\left(E^{\mathrm{T}} \dot{d}-J(a) E^{\mathrm{T}} d\right)+J(a) \tilde{M} E^{\mathrm{T}} d+J\left(E^{\mathrm{T}} d\right) \tilde{M} e .
$$

This is linear in the parameter errors, $\tilde{M}$, so we will write instead,

$$
n(t)=H(E, e, a, d, \dot{d}) p,
$$

where $H$ is a known matrix valued function of available data, and $p$ is a vector of unknown inertia parameter errors.

The reflex procedure in linear-in-parameter adaptive control theory is to now adaptively adjust $p$ according to the rule

$$
\dot{p}=-H^{\mathrm{T}}\left[0, M^{-1}\right](D \vartheta)^{\mathrm{T}},
$$

where $\vartheta$ is a strict Lyapunov function for the homogeneous plant (6) (the array of partial derivatives of a function, $f$, with respect to the specified coordinates is denoted $D f$ ), since this leads to a closed loop system of the form

$$
\begin{aligned}
& {\left[\begin{array}{c}
\dot{E} \\
\dot{e}
\end{array}\right]=\left[\begin{array}{c}
E J(e) \\
-M^{-1}\left[J(e) M e+K_{2} e+\operatorname{grad} \varphi(E)\right]
\end{array}\right]+\left[\begin{array}{c}
0 \\
M^{-1}
\end{array}\right] H p} \\
& \dot{p}=-H^{\mathrm{T}}\left[0, M^{-1}\right](D \vartheta)^{\mathrm{T}} \text {, }
\end{aligned}
$$

for which a Lyapunov function is

$$
\vartheta+\frac{1}{2} p^{\mathrm{T}} p
$$

But this is absurd on face value, since we have already assumed that the actual value of $M$ is unknown and cannot be used in (12).

Now examine the particular form of $\vartheta$ in the present situation,

$$
\begin{aligned}
\vartheta & =\gamma \circ \eta+\alpha \\
& =\gamma \circ\left(\frac{1}{2} e^{\mathrm{T}} M e+\varphi(E)\right)+e^{\mathrm{T}} M(F d \varphi(E)),
\end{aligned}
$$

and observe that

$D \vartheta\left[\begin{array}{c}0 \\ M^{-1}\end{array}\right]=[\partial \vartheta / \partial E, \partial \vartheta / \partial e]\left[\begin{array}{c}0 \\ M^{-1}\end{array}\right]=\left(\gamma^{\prime} \circ \eta\right) e^{\mathrm{T}}+(F d \varphi(E))^{\mathrm{T}}$.

Clearly, if $\vartheta$ has unscaled energy, $\gamma(\chi)=\bar{\gamma} \chi$, then this expression is free of dependence upon $M$, and it follows that $(-12)$ is readily realizable. Of course, Proposition 3.2 provides a recipe for constructing a strict Lyapunov function with unscaled energy for (1) provided that $\varphi$ is a "uniform" potential in the sense defined above.

Lemma 3.3 The modified trace function (3) is a uniform potential on $\mathrm{SO}(3)$.

Proof: It is shown in [8] that $\lambda_{\varphi}=\frac{1}{2} \nu_{P} \xi$, is a lower comparison function for $\varphi$, and

$$
v_{d \varphi}^{2}=\left[\mu_{P}^{2}+\mu_{P}^{2}\right] \xi,
$$

is an upper comparison function for $\|d \varphi\|, \xi$ having been defined in Proposition 2.1. It follows that

$$
v_{d \varphi}^{2} \circ \lambda_{\varphi}(\chi)=2 \frac{\left[\mu_{P}^{2}+\mu_{P}^{2}\right]}{\nu_{P}} \chi
$$

is a multiple of the identity element, $1 \mathcal{K}_{r}$. Moreover, since $S O(3)$ is compact, the hessian of $\varphi$, being smooth, has a bounded norm and the second derivative to first derivative ratio in the definition of a uniform potential (10) must be bounded as well.

We may now apply Proposition 3.2 and define a morphism $F$, according to the construction (11) to get

$$
F: T_{R}^{*} S O(3) \rightarrow T_{R} S O(3): w^{\mathrm{T}} \mapsto \frac{1}{1+\|d \varphi(R)\|} w .
$$

This yields the angle map

$$
\alpha(E, e)=\frac{2}{1+2\left\|J^{\dagger}(P E)\right\|} e^{\mathrm{T}} M J^{\dagger}(P E),
$$

following the prescription (7). The complete Lyapnov function is now given as

$$
\vartheta(E, e)=\bar{\gamma}\left(e^{\mathrm{T}} M e+\operatorname{tr}\{P E\}\right)+\frac{2}{1+2\left\|J^{\dagger}(P E)\right\|} e^{\mathrm{T}} M J^{\dagger}(P E),
$$

where the constant,

$$
\bar{\gamma}>\max \left\{1+\overline{\mu_{M}} \sigma_{K_{2}}, \sigma_{M} / 4,1 / \overline{\nu_{K_{2}}}\right\} ; \quad \sigma_{A} \triangleq \frac{\overline{\mu_{A}}}{\overline{\nu_{A}}},
$$

is chosen to satisfy the inequalities (9). The adaptive law, (12),

$$
\dot{p}=-H^{\mathrm{T}}\left(\bar{\gamma} e+\frac{2}{1+2\left\|J^{\dagger}(P E)\right\|} J^{\dagger}(P E)\right),
$$

is clearly realizable with no knowledge of $M$ except an upper bound on its largest magnitude eigenvalue and a smallest bound on its smallest magnitude eigenvalue required in the definition of $\bar{\gamma}$.

Theorem 4 The adaptive procedure (16) results in a stable closed loop state-parameter error system (13). If the reference signals, $D, d, \dot{d}$, are bounded then the rotation error, $E$, tends asymptotically toward one of the four critical points of $\varphi$ with zero velocity error, $e=0$. 
Proof:

The positive definite Lyapunov function $\vartheta+\frac{1}{2} p^{\mathrm{T}} p$ has the derivative $L_{f}(\vartheta)$ along trajectories of the full closed loop adaptive system (13), and this is bounded above by a negative definite quadratic form, $L_{f}(\vartheta) \leq-x^{\mathrm{T}} Q(E) x, x=$ $[\|d \varphi(E)\|,\|e\| \|$, according to Theorem 3 . This demonstrates the stability of the closed loop system.

Since $S O(3)$ is compact, $Q(E)$ admits a non-zero constant lower bound, $\overline{\nu_{Q}}$, so that $L_{f}(\vartheta) \leq-\overline{\nu_{Q}} x^{\mathrm{T}} x<0$. Since $L_{f}(\vartheta)$ is the derivative of a scalar function which remains bounded for all time, we have

$$
\infty>\int_{0}^{\infty} L_{f}(\vartheta)(t) d t>\int_{0}^{\infty} \overline{\nu_{Q}} x^{\mathrm{T}} x d t .
$$

Thus $x$ is an $\mathcal{L}^{2}$ function of time. Now $\dot{x}$ is governed by $(\dot{E}, \dot{e})$, which is bounded as long as the reference signals, $(D, d, \dot{d})$ are bounded. Since an $\mathcal{L}^{2}$ function with bounded derivative tends to zero [13], the boundedness of the reference signals ensures to convergence of $e$ and $d \varphi$ to zero. Since there are only four critical points of $\varphi$ in $S O(3)$ according to Lemma 2.3 , the conclusion follows.

\section{Acknowledgements}

Discussions with Profs. C. Byrnes, W. Massey, J. Pierce, and E. Sontag have been most helpful in pointing the author toward references to a variety of technical results used in this paper. Profs. A.S. Morse and K. S. Narendra have lent critical advice and patient ears.

\section{A Comparison Functions}

$$
\begin{aligned}
& \text { Given two real open intervals, } \mathcal{I}_{1}, \mathcal{I}_{2} \subset \mathbb{R} \text {, define the group of com- } \\
& \text { parison functions, } \\
& \mathcal{K}_{r}\left[\mathcal{I}_{1}, \mathcal{I}_{2}\right] \triangleq\left\{\kappa \in C^{r}\left[\mathcal{I}_{1}, I_{2}\right]: \lim _{t \rightarrow \inf I_{1}} \kappa(t)=\inf \mathcal{I}_{2} \text { and } t_{1}<t_{2} \Rightarrow \kappa\left(t_{1}\right)<\kappa\left(t_{2}\right)\right\} \text {, } \\
& \text { to be those } C^{r} \text { diffeomorphisms between the two intervals that take } \\
& \text { the lower boundary point of } \mathcal{I}_{1} \text { to the lower boundary point of } \mathcal{I}_{2} \text {. } \\
& \tilde{\kappa}(x) \triangleq \kappa \circ \rho\left(x_{d}, x\right), \\
& \lambda_{\psi} \circ \rho(\mathcal{B}, x) \leq \psi(x) \leq v_{\psi} \circ \rho(\mathcal{B}, x) \\
& \rho(\mathcal{B}, x) \triangleq \inf _{y \in \mathcal{B}} \rho(y, x)
\end{aligned}
$$

Now assume that the metric, $\rho$, is induced by some Riemannian metric, $\|\cdot\|$. This induces a metric on every vector bundle, $\nu$, ove $\mathcal{X}$, so that the magnitude of any morphism, $M \in \mathcal{M}^{\top}\left[\mathcal{V}_{1}, \mathcal{V}_{2}\right]$, may be well defined. For our purposes this is best accomplished by recourse to the scalar valued maps $\nu_{M}, \mu_{M} \in C^{r}\left[\mathcal{X}, \overline{\mathbb{R}^{+}}\right]$,

$\mu_{M}(x) \triangleq \sup \left\{\|M v\|_{v_{2}}: v \in\left(\mathcal{V}_{1}\right)_{x}\right.$ and $\left.\|v\|_{\nu_{1}}=1\right\}$

$\nu_{M}(x) \triangleq \inf \left\{\|M v\|_{\nu_{2}}: v \in\left(\mathcal{V}_{1}\right)_{x}\right.$ and $\left.\|v\| \nu_{1}=1\right\}$

If a compact "reference set" of interest, $B \subset \mathcal{X}$ has been chosen, then these upper and lower magnitude maps generate a non-decreasing scalar function,

and a non-increasing scalar function,

$$
v_{M}(\chi) \triangleq \sup _{\rho(B, x) \leq x} \mu_{M}(x),
$$

$$
\lambda_{M}(\chi) \triangleq \inf _{p(B, x) \leq \chi} \nu_{M}(x)
$$

which may be thought of as comparison functions for $\mu_{M}, \nu_{M}$, but, of course, are not in $\mathcal{K}_{r}\left[\mathcal{I}_{1},\right]$ since they may not be invertible (and, even if invertible, the second function is monotone in the wrong direction)

Finally, denote the largest and smallest magnitude of $M$ by

$$
\overline{\mu_{M}} \triangleq v_{M}\left(\sup I_{1}\right) ; \quad \overline{\bar{\nu}_{M}} \triangleq \lambda_{M}\left(\sup I_{1}\right)
$$

\section{References}

[1] Ralph Abraham and Jerrold E. Marsden. Foundations of Me chanics. Benjamin/Cummings, Reading, MA, 1978.

[2] V. I. Arnold. Mathematical Methods of Classical Mechanics. Springer-Verlag, N.Y., 1978.

[3] N. P. Bhatia and G. P. Szegö . Dynamical Systems: Stability Theory and Applications. Springer-Verlag, Berlin, 1967.

[4] D. R. J. Chillingworth, J. E. Marsden, and Y.H. Wan. Symmetry and bifurcation in three-dimensional elasticity, Part I. Arch. Rat. Mech. Anal., 80(4):295-331, 1982.

[5] Theodore Frankel. Critical submanifolds and stiefel manifolds. In Stewart S. Cairns, editor, Differential and Combinatorial Topology, pages 37-54, Princeton University, Princeton, 1965.

[6] Wolfgang Hahn. Stability of Motion. Springer-Verlag, New York, 1967.

[7] Morris W. Hirsch. Differential Topology. Springer-Verlag, NY, 1976.

[8] D. E. Koditschek. Application of a New Lyapunov Function: Global Adaptive Inverse Dynamics for a Single Rigid Body. Technical Report 8806, Center for Systems Science, Yale University, Sept. 1988 .

[9] D. E. Koditschek. A Strict Global Lyapunov Function for Mechanical Systems. Technical Report 8707, Center for Systems Science, Yale University, November 1987.

[10] Daniel E. Koditschek. Strict global lyapunov functions for me chanical systems. In Proc. American Control Conference, American Automatic Control Council, Atlanta, GA., Jun 1988.

[11] George Meyer. Design and Global Analysis of Spacecraft Attitude Control Systems. NASA Technical Report TR R-361, Ames Research Center, Moffett Field, CA, Mar 1971.

[12] John W. Milnor. Topology from the Differentiable Viewpoint. The University Press of Virginia, Charlottesville, Va., 1965.

[13] K. S. Narendra and A. Annaswamy. Stable Adaptive Systems. Prentice-Hall, NY, 1988.

[14] Sir W. Thompson and P. G. Tait. Treatise on Natural Philosophy. University of Cambridge Press, 1886, Cambridge. 